\title{
A fast method for determination of creep forces in non-Hertzian contact of wheel and rail based on a book of tables
}

\author{
Jerzy Piotrowski ${ }^{1}$, Stefano Bruni ${ }^{2}$, Binbin Liu ${ }^{2}$, Egidio Di Gialleonardo ${ }^{2}$ \\ ${ }^{1}$ Institute of Vehicles, Warsaw University of Technology, Narbutta 84, 02-524 Warsaw, Poland \\ ${ }^{2}$ Dipartimento di Meccanica, Politecnico di Milano, Via La Masa 1, Milano 20156, Italy
}

\section{ORCID}

Jerzy Piotrowski: 0000-0002-7981-7552

Stefano Bruni: 0000-0003-2177-5254

Binbin Liu: 0000-0003-2482-8729

Egidio Di Gialleonardo: 0000-0003-1393-2942

Corresponding author: Jerzy Piotrowski ( jpt@ simr.pw.edu.pl)

Address for correspondence:

Jerzy Piotrowski

Institute of Vehicles

Warsaw University of Technology

Narbutta 84

02-524 Warsaw, Poland 


\begin{abstract}
The Kalker book of tables for non-Hertzian contact described in a previous work from the Authors provides creep forces but the moment generated in the contact patch is not available. The extended Kalker book of tables for non-Hertzian contact (KBTNH) presented in this paper provides creep forces as well as the moment. The paper presents the simplified linear theory of rolling contact for a simple double-elliptical contact (SDEC) region used for regularisation of contact patches and resulting from this theory the full symmetry relations for creep forces and moment. The parameterisation of variables and structure of the extended book of tables are described. The extended Kalker book of tables of moderate volume has been computed in co-simulation Matlab-CONTACT. The creep forces and moment obtained from KBTNH have been compared to those obtained directly by program CONTACT and FASTSIM algorithm. The comparison shows that KBTNH is in good agreement with CONTACT for a wide range of creepage conditions and shapes of the contact patch, whereas the use of FASTSIM may lead to significant deviations from the reference CONTACT solutions. The presented example of application is a realistic case of a freight wagon curving simulation, where KBTNH has been used to assess the contribution of the moment into the unit frictional work dissipated in contacts of wheels and rails. The high calculation speed and good accuracy of determining creep forces for non-Hertzian wheel-rail contact make the proposed method a suitable tool for multibody system (MBS) simulation programs oriented for rail vehicle dynamics.
\end{abstract}

Keywords: wheel-rail contact, creep force, non-Hertzian contact, book of tables, rail vehicle dynamics, FASTSIM

\title{
1. INTRODUCTION
}

The determination of creep forces at the wheel-rail interface in MBS simulations mostly rely on the use of the FASTSIM algorithm [?] or on specific adoptions of this method for the applications of non-Hertzian contact conditions. However, the use of FASTSIM for a nonHertzian patch poses problems in terms of defining the value of the elasticity parameter(s). As an alternative to FASTSIM, FaStrip was developed in [?] for creep force estimation where the strip theory has been extended from the two-dimensional rolling contact solution to threedimensional contact with combination of FASTSIM to improve the accuracy of the tangential stress distribution and the creep force estimation with respect to the original FASTSIM. The well-known program CONTACT [?] is still not widely used in MBS simulations, due to the large computational effort required.

Therefore, a fast method for determination of creep forces in non-Hertzian contact of wheel and rail is highly desirable. To this end, a new regularisation of the non-elliptical contact patch has been introduced in the work [1], which enabled building the look-up table called the Kalker book of tables for non-Hertzian contact (KBTNH). The Kalker book of tables is a fast creep force generator that can be used by MBS simulation programs. The nonelliptical contact patch is regularised by a simple double-elliptical contact region (SDEC) [1]. The SDEC region is especially suitable for regularisation of contact patches obtained with approximate non-Hertzian methods for solving the normal contact problem of wheel and rail. The new regularisation is suitable for wheels and rails with nominal or measured profiles, although in its present form the method cannot be applied to conformal contact situations .

The paper [1] describes the procedure of regularisation of the non-elliptical contact patch, 
the structure of the Kalker book of tables, the parameterisation of the independent variables and the creep forces.

In the work [1] a moderate volume Kalker book of tables for SDEC region has been computed in co-simulation of Matlab and program CONTACT [2] by solving the normal contact problems for a number of SDEC contact regions and calculating creep forces for prescribed creepages. The tables provide the lateral and longitudinal creep forces but the moment generated in the contact patch is not available. It means that the distribution of tangential stress over the contact patch is replaced by the resultant creep forces in lateral and longitudinal direction only.

The influence of moment in the contact patch is not of primary importance for the dynamics of the classical running gear because the arms of action of creep forces over wheelset are much larger than the arms of forces producing the moment in the contact patch. This is why this term is often neglected by software packages commonly used in railway vehicle dynamics simulation. However, according to [3], neglecting the moment component leads to incoherent results and the contribution of the moment to the frictional work dissipated in contact has been found significant in the cases studied in which the equilibrium situation when imposing a certain lateral shift to all the wheelsets and the quasi-static equilibrium during curve negotiation of a conventional bogie. Furthermore, the influence of moment should be considered for unconventional running gear designs with independent wheels that may rotate and yaw independently since in this case its contribution would not be negligible anymore.

When this work was firstly presented during the EUROMECH Colloquium 578 on Rolling Contact in Multibody Dynamics, it generated a debate on the actual influence of spin on longitudinal creep forces for non-symmetric contact patches as those that can be represented by the SDEC approximation. It was argued that spin cannot generate the longitudinal creep force in non-elliptic, asymmetric contact patches, similarly as it does not in elliptic (symmetric) contact patches. In order to gain more insight and experimental evidence in this topic, an experiment was devised to show quantitatively this effect and a description of the experimental setup and of the results obtained is included in this paper.

Therefore, the aim of the present work is threefold:

1) to extend the KBTNH by including the moment as one additional tabulated quantity and to derive the proper symmetry relations for this quantity. This extension of the $\mathrm{KBTNH}$ enables the efficient and reliable estimation of moment and the frictional work dissipated in the wheel/rail contact interface during vehicle dynamics simulation process;

2) to quantify the accuracy of the $\mathrm{KBTNH}$ to estimate the moment compared to CONTACT as the reference solution and to FASTSIM as an alternative fast approximate solution;

3) to present results from an experimental set-up, providing evidence of the effect of spin on longitudinal creep forces for a non-elliptical contact patch of SDEC shape.

\section{LINEAR SIMPLIFIED THEORY FOR SDEC CONTACT REGION}

The SDEC region is presented in Figure 1. It is described by the following equations [1]:

$$
\text { If } y>0 \quad \text { then } \frac{x^{2}}{a^{2}}+\frac{y^{2}}{b^{2}(1+\psi)^{2}}=1 \text {. }
$$

The leading edge $\mathrm{x}$ co-ordinate of the SDEC region for $\mathrm{y}>0$ is 


$$
\begin{aligned}
& \text { If } y<=0 \quad x_{l p}(y)=a \sqrt{1-\frac{y^{2}}{b^{2}(1+\psi)^{2}}} . \\
& \text { then } \frac{x^{2}}{a^{2}}+\frac{y^{2}}{b^{2}(1-\psi)^{2}}=1 .
\end{aligned}
$$

The leading edge $\mathrm{x}$ co-ordinate of the SDEC region for $\mathrm{y} \leq 0$ is

$$
x_{\ln }(y)=a \sqrt{1-\frac{y^{2}}{b^{2}(1-\psi)^{2}}} .
$$

The parameter $\psi$ is called the "shape number" of the SDEC contact patch. It is defined as

$$
\psi=\mathrm{y}_{0} / \mathrm{b} .
$$

\section{FIGURE 1 TO APPEAR ABOUT HERE}

According to Kalker's simplified linear theory, the tangential traction distribution is derived in the following form:

$$
\begin{aligned}
& \mathrm{p}_{\mathrm{x}}=\frac{1}{\mathrm{~L}}\left(\mathrm{x}-\mathrm{x}_{1}\right)\left(v_{\mathrm{x}}-\mathrm{y} \varphi\right), \\
& \mathrm{p}_{\mathrm{y}}=\frac{1}{\mathrm{~L}}\left[\left(\mathrm{x}-\mathrm{x}_{1}\right) v_{\mathrm{y}}+\frac{1}{2}\left(\mathrm{x}^{2}-\mathrm{x}_{1}^{2}\right) \varphi\right] .
\end{aligned}
$$

The creep forces $F_{x}, F_{y}$ and moment $M_{z}$ are obtained by integration of the tangential traction $\mathrm{p}_{\mathrm{x}}, \mathrm{p}_{\mathrm{y}}$ over the contact patch (in the equations below, subscripts " $\mathrm{n}$ " and " $\mathrm{p}$ " stand for negative and positive $y$, respectively).

$$
\begin{aligned}
F_{x} & =\frac{1}{L} \int_{-b+y_{0}}^{0} \int_{-x_{l n}}^{x_{\ln }}\left(x-x_{\ln }\right)\left(v_{x}-y \varphi\right) d x d y+\frac{1}{L} \int_{0}^{b+y_{0}} \int_{-x_{l p}}^{x_{l p}}\left(x-x_{l p}\right)\left(v_{x}-y \varphi\right) d x d y= \\
& =-\frac{8}{3 L} a^{2} b v_{x}+\frac{2}{L} a^{2} b y_{0} \varphi=-\frac{8}{3 L} a^{2} b v_{x}+\frac{2}{L} a^{2} b^{2} \psi \varphi,
\end{aligned}
$$




$$
\begin{aligned}
& \mathrm{F}_{\mathrm{y}}=\frac{1}{\mathrm{~L}} \int_{-\mathrm{b}+\mathrm{y}_{0}-\mathrm{x}_{\ln }}^{0} \int_{\ln }^{\mathrm{x}_{\ln }}\left[\left(\mathrm{x}-\mathrm{x}_{\ln }\right) v_{\mathrm{y}}+\frac{1}{2}\left(\mathrm{x}^{2}-\mathrm{x}_{\ln }^{2}\right) \varphi\right] \mathrm{dxdy}+ \\
& +\frac{1}{\mathrm{~L}} \int_{0}^{\mathrm{b}+\mathrm{y}_{0}} \int_{-\mathrm{x}_{\mathrm{lp}}}^{\mathrm{x}_{\mathrm{lp}}}\left[\left(\mathrm{x}-\mathrm{x}_{\mathrm{lp}}\right) v_{\mathrm{y}}+\frac{1}{2}\left(\mathrm{x}^{2}-\mathrm{x}_{\mathrm{lp}}^{2}\right) \varphi\right] \mathrm{dxdy}=-\frac{8}{3 \mathrm{~L}} \mathrm{a}^{2} \mathrm{~b} v_{\mathrm{y}}-\frac{\pi}{4 \mathrm{~L}} \mathrm{a}^{3} \mathrm{~b} \varphi . \\
& \mathrm{M}_{\mathrm{z}}=\int_{-\mathrm{b}+\mathrm{y}_{0}-\mathrm{x}_{\ln }}^{0} \int_{\mathrm{ln}}\left(\mathrm{x} \cdot \mathrm{p}_{\mathrm{yn}}-\mathrm{y} \cdot \mathrm{p}_{\mathrm{xn}}\right) \mathrm{dxdy}+\int_{0}^{\mathrm{b}+\mathrm{y}_{0}} \int_{-\mathrm{x}_{\mathrm{lp}}}^{\mathrm{x}_{\mathrm{lp}}}\left(\mathrm{x} \cdot \mathrm{p}_{\mathrm{yp}}-\mathrm{y} \cdot \mathrm{p}_{\mathrm{xp}}\right) \mathrm{dxdy}= \\
& =\frac{2}{\mathrm{~L}} \mathrm{a}^{2} \mathrm{~b}^{2} \psi v_{\mathrm{x}}+\frac{\pi}{4 \mathrm{~L}} \mathrm{a}^{3} \mathrm{~b} v_{\mathrm{y}}-\frac{8}{15 \mathrm{~L}} \mathrm{a}^{2} \mathrm{~b}^{3}\left(1+3 \psi^{2}\right) \varphi .
\end{aligned}
$$

where

$$
\mathrm{p}_{\mathrm{xj}}=\frac{1}{\mathrm{~L}}\left(\mathrm{x}-\mathrm{x}_{\mathrm{lj}}\right)\left(v_{\mathrm{x}}-\mathrm{y} \varphi\right), \quad \mathrm{p}_{\mathrm{yj}}=\frac{1}{\mathrm{~L}}\left[\left(\mathrm{x}-\mathrm{x}_{\mathrm{lj}}\right) v_{\mathrm{y}}+\frac{1}{2}\left(\mathrm{x}^{2}-\mathrm{x}_{\mathrm{lj}}^{2}\right) \varphi\right], \quad(\mathrm{j}=\mathrm{n}, \mathrm{p}) .
$$

\section{THE PARAMETERISATION OF CREEPAGES AND CREEP FORCES}

The variables involved in the definition of the book of tables are the creepages, creep forces and moment. These quantities are parameterised in order to ensure sufficiently broad ranges of outputs for application in MBS simulation programs.

After [1] we introduce the following parameterisation of creepages:

$$
\xi=\frac{\rho v_{\mathrm{x}}}{\mu \mathrm{c}}, \quad \eta=\frac{\rho v_{\mathrm{y}}}{\mu \mathrm{c}}, \quad \chi=\frac{\rho \varphi}{\mu},
$$

where:

$\mathrm{c}=\sqrt{\mathrm{a} \cdot \mathrm{b}}$ - equivalent radius of the SDEC region,

$$
\rho=\frac{4 \mathrm{E}}{3\left(1-\sigma^{2}\right) \mathrm{N}}\left(\frac{\mathrm{A}}{\pi \mathrm{n}_{\mathrm{a}} \mathrm{n}_{\mathrm{b}}}\right)^{3 / 2} \text { - characteristic length of the elliptical contact patch }
$$

with semi axes $\mathrm{a}, \mathrm{b}$ and area $\mathrm{A}$ that is formed under normal load N. E is Young's modulus, $\mathrm{n}_{\mathrm{a}}, \mathrm{n}_{\mathrm{b}}$ are tabulated coefficients as functions of $\theta=\max (\mathrm{a} / \mathrm{b}, \mathrm{b} / \mathrm{a})[4], \sigma-$ Poisson ratio, $\mu$ coefficient of friction.

The product $\mathrm{n}_{\mathrm{a}} \cdot \mathrm{n}_{\mathrm{b}}$ can be calculated by the empirical formula [1]

$$
\mathrm{n}_{\mathrm{a}} \mathrm{n}_{\mathrm{b}}=\mathrm{a} \theta^{3}+\mathrm{b} \theta^{2}+\mathrm{c} \theta+\mathrm{d}
$$

where: $\mathrm{a}=3.618652 \times 10^{-5}, \mathrm{~b}=-2.687505 \times 10^{-3}, \mathrm{c}=9.5658 \times 10^{-2}, \mathrm{~d}=0.8872736$.

The non-dimensional creep forces and moment are defined as follows:

$$
\mathrm{f}_{\mathrm{x}}=\frac{\mathrm{F}_{\mathrm{x}}}{\mu \mathrm{N}}, \quad \mathrm{f}_{\mathrm{y}}=\frac{\mathrm{F}_{\mathrm{y}}}{\mu \mathrm{N}}, \quad \mathrm{m}_{\mathrm{z}}=\frac{\mathrm{M}_{\mathrm{z}}}{\mu \mathrm{cN}}
$$


With introduced parameters the book of tables will have five independent variables $\xi, \eta, \chi, \psi, g=a / b$ and three output quantities $\mathrm{f}_{\mathrm{x}}, \mathrm{f}_{\mathrm{y}}$ and $\mathrm{m}_{\mathrm{z}}$.

Now the new independent variables $v$ and $\alpha$ replacing $\xi$ and $\eta$ are introduced [1].

$$
\begin{gathered}
v=\sqrt{\xi^{2}+\eta^{2}}, \\
\text { if } \xi \neq 0, \quad \alpha=\operatorname{arctg} \frac{\eta}{\xi},
\end{gathered}
$$

else

$$
\alpha=\left\{\begin{array}{cccc}
\pi / 2 & \text { if } & \eta>0 \\
0 & \text { if } & & \eta=0 \\
-\pi / 2 & \text { if } & \eta<0
\end{array}\right.
$$

The book of tables will have now five new independent variables $v, \alpha, \chi, \psi, g$.

The normalised creep forces $\mathrm{f}_{\mathrm{x}}, \mathrm{f}_{\mathrm{y}}$ and moment $\mathrm{m}_{\mathrm{z}}$ are functions of these variables.

$$
\begin{gathered}
\mathrm{f}_{\mathrm{x}}(v, \alpha, \chi, \psi, \mathrm{g})=\frac{\mathrm{F}_{\mathrm{x}}}{\mu \mathrm{N}} \\
\mathrm{f}_{\mathrm{y}}(v, \alpha, \chi, \psi, \mathrm{g})=\frac{\mathrm{F}_{\mathrm{y}}}{\mu \mathrm{N}} \\
\mathrm{m}_{\mathrm{z}}(v, \alpha, \chi, \psi, \mathrm{g})=\frac{\mathrm{M}_{\mathrm{z}}}{\mu \mathrm{cN}}
\end{gathered}
$$

\section{THE STRUCTURE AND VOLUME OF THE EXTENDED KALKER BOOK OF TABLES}

It is proposed to build a moderate volume book of tables suitable for modern running gears where the vehicle is guided by the track without severe contact of flanges. For this purpose, a table with relatively small range of spin parameter is sufficient and with not very elongated shapes of the SDEC region. We propose the following set of values:

$$
\begin{aligned}
& \chi=0,0.25,0.5,0.75,1.0,1.33,1.66,2.0 \quad\left(\mathrm{n}_{\chi}=8\right) \\
& \mathrm{g}=\mathrm{a} / \mathrm{b}=0.2,0.5,0.75,1,2,3,4,5 \quad\left(\mathrm{n}_{\mathrm{g}}=8\right) \\
& \alpha=-\frac{\pi}{2},-\frac{\pi}{3},-\frac{\pi}{6}, 0, \frac{\pi}{6}, \frac{\pi}{3}, \frac{\pi}{2}, \quad\left(\mathrm{n}_{\alpha}=7\right) \\
& v=0.0,0.1,0.2,0.3,0.4,0.5,0.7,0.9,1.1,1.4,1.7,2.0,2.5,3.0,3.5,4.0,5.0,6.0 \text {, } \\
& \text { 7.0, 8.0, 9.0, 10.0, } 12.0 \quad\left(\mathrm{n}_{v}=23\right) \\
& \psi=-0.9,-0.8,-0.6,-0.4,-0.2,0.0,0.2,0.4,0.6,0.8,0.9\left(\mathrm{n}_{\psi}=11\right)
\end{aligned}
$$

The range of the $\mathrm{a} / \mathrm{b}$ ratio considered in the construction of the table reflects the intention to obtain a book of tables of moderate volume which is suitable for running conditions in 
which severe flanging is not reached. However, the same methodology could be applied to obtain a more general book of tables, at the expense of a larger volume. The discretisation of the spin parameter $\chi$ is slightly different from that in [1]. The number of values is the same but spin is more homogenous in the range $0-2$.

This choice results in:

- number of runs of CONTACT program $\mathrm{N}_{\mathrm{c}}=\mathrm{n}_{\chi} \mathrm{n}_{\mathrm{g}} \mathrm{n}_{\alpha} \mathrm{n}_{v} \mathrm{n}_{\psi}=113344$,

- $\quad$ number of resulting data numbers $\mathrm{N}_{\mathrm{d}}=3 \mathrm{~N}_{\mathrm{c}}=3 \cdot 113344=340032$,

- $\quad$ volume of the data set $\mathrm{N}_{\mathrm{s}}=4 \mathrm{~N}_{\mathrm{d}}=4 \cdot 340032=1360128 \mathrm{~B}=1.36 \mathrm{MB}$.

It is worth mentioning that the normal pressure distribution on any SDEC contact region with $\psi \neq 0$ is similar to that shown in Figure 2(c). It has a distinct summit at the geometrical point of contact. Its corresponding contact patch shape and normal pressure profile at $\mathrm{y}=0$ are shown in Figures 2(a) and 2(b), respectively. In each case during generating KBTNH the real traction bound was taken into account by iteratively solving the normal contact problem to obtain the SDEC contact patch. The details are described in [1]. It is worth mentioning that KBTNH is for non-conformal contact in its current version, similarly as USETAB is for elliptical contact satisfying Hertz's assumptions.

\section{FIGURE 2 TO APPEAR ABOUT HERE}

\section{THE SYMMETRY RELATIONS FOR CREEP FORCES AND MOMENT}

According to Equations (13-15) and discretisation of the spin parameter $\chi$, two of the initial independent variables, namely the longitudinal creepage parameter $\xi$ and spin parameter $\chi$ have only non-negative values. To evaluate the creep forces and moment for negative values of these parameters, the symmetry relations for the creep forces and moment are necessary.

The rolling law of the simplified linear theory for SDEC (6), (7), (8) can be rewritten to the form

$$
\begin{aligned}
& \mathrm{f}_{\mathrm{x}}=-\mathrm{c}_{\mathrm{xx}} \xi+\mathrm{c}_{\mathrm{xz}} \psi \chi, \\
& \mathrm{f}_{\mathrm{y}}=-\mathrm{c}_{\mathrm{yy}} \eta-\mathrm{c}_{\mathrm{yz}} \chi, \\
& \mathrm{m}_{\mathrm{z}}=\mathrm{c}_{\mathrm{zx}} \psi v_{\mathrm{x}}+\mathrm{c}_{\mathrm{zy}} \eta-\mathrm{c}_{\mathrm{zz}} \chi
\end{aligned}
$$

where

$$
\begin{aligned}
& c_{x x}=c_{y y}=\frac{8}{3 N L \rho} \cdot \sqrt{a^{5} b^{3}}, c_{x z}=c_{z x}=\frac{2}{N L \rho} a^{2} b^{2}, c_{y z}=c_{z y}=\frac{\pi}{4 N L \rho} a^{3} b, \\
& c_{z z}=\frac{8}{15 N L \rho}\left(1+3 \psi^{2}\right) \sqrt{a^{3} b^{5}} .
\end{aligned}
$$

TABLE 1 TO APPEAR ABOUT HERE 
Formulae (19-21) are used to determine the symmetry relations shown in Table 1. Table 1 shows only those relations where $\mathrm{f}_{\mathrm{x}}, \mathrm{f}_{\mathrm{y}}, \mathrm{m}_{\mathrm{z}}$ are negated due to negation of $\xi$ and/or $\chi$.

\section{EVALUATION OF THE KBTNH MODEL}

\subsection{Pure spin condition}

The performance of KBTNH in calculation of the tangential creep forces has been described in [1], while the calculation and the evaluation of its performance in terms of moment produced in the contact area is missing. It is the aim of this section to complete the evaluation and to extend to the comparison between KBTNH and elliptical contact.

Two separate groups of comparisons are considered for the evaluation of the moment calculated by KBTNH:

- Comparison with FASTSIM applied to the equivalent elliptic contact of SDEC region and comparison with CONTACT.

- Comparison with another version of FASTSIM as used by K-P approximate method [5].

In the first comparison the characteristic length $\rho$ is constant for given constant semi-axis ratio $g$ and any shape number $\psi$ for CONTACT and for KBTNH. This is due to the fundamental property of SDEC, which is that if SDEC contact region with parameters a, b, $\psi$ is the result of the normal contact problem solution obtained by CONTACT for given penetration, then the normal load is the same as for the Hertzian contact ellipse with the semi-axes a, b obtained for the same penetration [1].

In contrast, K-P method being an approximate one gives different normal loads for constant $\mathrm{g}=\mathrm{a} / \mathrm{b}$ and various $\psi$ and, according to Equation (11), the characteristic length varies for given constant $\mathrm{g}$ and different shape numbers $\psi$. For adequate comparison, the same characteristic length that used in K-P will be applied when KBTNH is called in the second comparison. Due to this configuration, the data of KBTNH shown in the plots for the two groups of comparison are slightly different.

As shown in Figure 3(a), the moments obtained by KBTNH and CONTACT agree well for all the cases studied, while FASTSIM is far from both. For the second kind of comparison shown in Figure 3(b), it can be concluded that for pure spin K-P does not predict the moment with sufficient accuracy. The maximum discrepancies with respect to KBTNH among all the cases considered are approximately $20 \%$. It also can be found that the discrepancies are influenced by the shape number and semi-axes ratio as they do for the creep forces [1].

FIGURE 3 TO APPEAR ABOUT HERE

\section{FIGURE 4 TO APPEAR ABOUT HERE}

Figure 4 compares again KBTNH, CONTACT; FASTSIM and KP for a single value of the shape number $\psi=0.4$ and for different values of spin. It can be observed from Figure $4(\mathrm{a})$ that KBTNH is fully in line with CONTACT for the given range of spin for all the cases considered. Regarding FASTSIM, significant discrepancies appear in the results of moment with an average discrepancy approximately $30 \%$ with reference to CONTACT. 
For the second kind of comparison shown in Figure 4(b), it can be found that considerable discrepancies exist in the moment in the entire range of spin considered in this study between K-P and KBTNH.

Figure 5 shows the same results as Figure 4 but this time for a different value of the shape factor $\psi=0.8$. Similar conclusions as obtained from Figure 4, while the discrepancies caused by FASTSIM with respect to CONTACT slightly increase with shape number, and the same applies to the comparison between K-P and KBTNH.

\section{FIGURE 5 TO APPEAR ABOUT HERE}

\subsection{Combined creepage and spin condition}

In order to check the accordance between KBTNH and CONTACT in terms of the output moment for realistic combined creepage and spin conditions the comparison has been made for $v_{\mathrm{y}} \equiv 0$ with such choice of $\varphi, v_{\mathrm{x}}$ that according to the Equation (8) of the linear theory $\mathrm{M}_{\mathrm{z}}=0$. Then

$$
\varphi=\frac{15}{4 b} \cdot \frac{\psi}{1+3 \psi^{2}} v_{x}
$$

or

$$
\chi=\frac{15}{4} \cdot \frac{\psi}{1+3 \psi^{2}} \sqrt{\mathrm{a} / \mathrm{b}} \cdot \xi .
$$

According to Equation (22), the following relations that obtained are shown in Table 2.

\section{TABLE 2 TO APPEAR ABOUT HERE}

The second case presented in Table 2 is chosen to show the comparisons for SDEC region with parameters $\mathrm{a}=4.2426 \mathrm{~mm}, \mathrm{~b}=8.4853 \mathrm{~mm}$, and penetration $\delta=0.1 \mathrm{~mm}$. The comparison results are plotted in Figure 6 (solid line with triangles for CONTACT and dashed line with stars for KBTNH). The normalised creep forces $f_{x}, f_{y}$ are also compared in the same figure for the sake of completeness.

\section{FIGURE 6 TO APPEAR ABOUT HERE}

It can be observed from Figure 6 that the contact solutions obtained by KBTNH and CONTACT are in good agreement. The comparison for the other cases listed in Table 2 has been performed to validate KBTNH with reference to CONTACT. However, only one case is shown here considering the space limitation in this paper.

Finally, in order to show how differ creep forces and moment for elliptical and nonelliptical contact, Figure 7 reports the comparisons of creep forces and moment from KBTNH $(\psi \neq 0)$ and elliptical KBTH $(\psi=0)$, respectively.

FIGURE 7 TO APPEAR ABOUT HERE 
It can be observed from Figure 7 that the difference between KBTNH and elliptical KBTH is significant in terms of the variation of the longitudinal force and moment with the modulus of creepage $v=\sqrt{\xi^{2}+\eta^{2}}$, whereas the lateral force is not so much influenced by the contact shape. Moreover, the magnitudes of the longitudinal creep force and moment obtained from KBTNH are considerable higher than those of elliptical contact (KBTH) under the same conditions, which may influence the MBS simulation results.

\section{EXAMPLE OF APPLICATION: CURVING SIMULATION OF A FREIGHT WAGON}

In the work [1] an example of application of KBTNH to curving simulation of a freight wagon has been presented using the curving program JPPGM [1] implementing the nonlinear curving theory [6]. In this paper we repeat the simulation of the same freight wagon but using extended KBTNH. The wheel/rail profile combination was KKVMZ/szynas60 (KKVMZ is a Czechoslovak profile [] and szynas60 is a Polish profile equivalent to UIC60) and the contact data for the profile combination have been calculated in [1] using K-P method [5]. For detailed parameters of the wagon that were used in the calculation please refer to [1].

The graphical output (see Figure 11 in [1]) of those calculations shows the series of contact patches for a set of lateral shifts of the wheelset against track centre line. For each wheelset position the value of the shape number $\psi$ have been calculated using SDEC regularisation. The results of contact calculations show that many contact patches resemble the SDEC region and the shape number $\psi$ for many positions of the wheelset takes substantial value.

The curving program was used in two ways the same as in [1] where the extended Kalker book of tables are used as the creep force generator in two modes with the shape number $\psi$ is constant zero and nonzero, respectively.

1. $\psi$ is set to 0 . The SDEC regularisation becomes the elliptical regularisation, which replaces non-elliptical contact patch with the equivalent ellipse. With $\psi=0, \mathrm{KBTNH}$ becomes the creep force generator for Hertzian rolling. We refer to this version as to KBTH.

2. To call creep forces from the extended Kalker book of tables the SDEC regularisation parameter $\psi$ is used. We will refer to this version as to KBTNH.

The presented results refer to $\mathrm{R}=460 \mathrm{~m}$ radius curve travelled by the vehicle with the cant deficiency of $1.9^{\circ}$. As the comparison between $\mathrm{KBTH}$ and $\mathrm{KBTBH}$ regarding the creepages and creep forces have been done in [1], the following comparison will concentrate on the moment and its related terms. The results are shown below in the form of the output tables from the program. They refer to the leading bogie of the wagon.

\section{TABLE 3 TO APPEAR ABOUT HERE}

The first column of Table 3 gives the wheel index (first number: 1-guiding wheelset, 2trailing wheelset, second number: 1-left, 2-right), see schematic illustration in Figure 8 . The $\mathrm{W}_{\mathrm{z}}$ column gives the unit frictional work $W_{z}=\left|v_{x} \cdot F_{x}+v_{y} \cdot F_{y}+\varphi \cdot M_{z}\right|$ in $[\mathrm{Nm} / \mathrm{m}]$, where $\mathrm{F}_{\mathrm{x}}$, $F_{y}$ and $M_{z}$ are the creep forces and the moment in the contact patch, and $v_{x}, v_{y}$ and $\varphi$ are creepages and spin. The MCON column shows the contribution of the moment to $\mathrm{W}_{\mathrm{Z}}$. The 
column with the header $\% \mathrm{~W}_{\mathrm{Z}}$ shows the percentage contribution of the moment to unit frictional work $\mathrm{W}_{\mathrm{Z}}$.

\section{FIGURE 8 TO APPEAR ABOUT HERE}

All results of simulations obtained with the use of KBTNH [1] and the present extended KBTNH are the same for the elliptical and SDEC regularisations, except for the unit frictional work $\mathrm{W}_{\mathrm{Z}}$, which is now calculated with the contribution of moment to the dissipation in the contact patch. This contribution, shown in column MCON in Table 3, is generally not high. For guiding wheels $(1,1)$ it is of the order of $10 \%$, but for wheels with low $\mathrm{W}_{\mathrm{Z}}$ it is higher and reaches $26 \%$.

\section{TESTS WITH EXPERIMENTAL APPARATUS}

In order to contribute with the experimental evidence to the debate on the influence of spin on longitudinal creep forces for non-elliptical, non-symmetric contact patches an experimental apparatus has been designed and built, and some initial results and information about this activity is given below.

\section{FIGURE 9 TO APPEAR ABOUT HERE}

Figure 9 shows a schematic drawing and a photo of the apparatus. It consists of a circular shield with two flanges, made of aluminum alloy, and two steel rollers that can roll over the flanges. The shield was machined on a high precision CNC milling machine. To enlarge contact patches and thus diminish the influence of the surface roughness the steel rollers are coated with a transparent polymer material. Each roller rotates freely around the axle. The profile of one of the flanges is composed of two arcs of circles that give non-elliptical contact patch very close to SDEC contact region (see Section 2). The other flange is profiled with a circular cross-section with constant radius that gives an elliptical contact patch.

The axle is guided relative to the shield by a pin in a hole at the center of the shield. There is a cylindrical joint connecting the pin with the axle, which ensures equal normal loads of the rollers. A tape with millimeter marks is glued to the roller. The indicator fixed to the axle allows measuring the angle of the roller rotation. The axle also has the indicator of its rotation in the form of a dial, which indicates the angle of the axle rotation, see Figure 9(b).

The dimensions of the apparatus are as follows (refer to Figure 9(a) for the meaning of symbols): $\mathrm{R}=90 \mathrm{~mm}, \mathrm{R}_{1}=120 \mathrm{~mm}, \mathrm{R}_{2}=15 \mathrm{~mm}, \mathrm{R}_{3}=20 \mathrm{~mm}, \mathrm{r}=20 \mathrm{~mm}$.

When the axle unit is rotated around the central pin then the rollers roll with spin due to angular velocity of the axle unit.

When the rollers are run over the side of the shield having circular profile an elliptic contact patch is formed and no longitudinal creep forces arise on the rollers. Hence, if in the first instance we neglect the drag in the bearings and rolling resistance, the relationship between the rotation $\alpha$ of the rollers and the rotation $\beta$ of the axle would be:

$$
\mathrm{r} \alpha=\mathrm{R} \beta \quad \rightarrow \quad \alpha=\frac{\mathrm{R}}{\mathrm{r}} \beta
$$

For given dimensions of the apparatus the ratio $2 \mathrm{R} / \mathrm{r}$ is an integer number. 
If the axle is rotated by exactly two turns (or multiples of this angle) the rollers will also rotate by an integer number of revolutions. If the experiment is started with the indicator on the roller at the " 0 " mark, at the end of the experiment the indicator will return at the " 0 " mark.

In reality, when this experiment is performed, the actual rotation of the rollers is lower due to occurrence of slight resistance to rotation of the roller that cannot be fully eliminated. We denote by $\Delta \alpha_{H}$ the remaining rotation angle of the roller to reach the " 0 " mark, when the axle is rotated by an integer multiple of two turns.

Next we rotate further the axle by such additional angle $\Delta \beta$ that the roller meets " 0 " mark. The angle $\Delta \beta$ determines the angular distance, which is lost by the roller due to drag in the bearings and rolling resistance.

The measured angles after 4 turns of the axle are: $\Delta \alpha_{\mathrm{H}}=1.584 \mathrm{rad}, \Delta \beta=20^{\circ}$.

Then, we repeat the experiment with the rollers making contact to the side of the flange with profile formed by two arcs of circles, giving rise to a non-elliptic, asymmetric contact patch. For this shape of the contact patch, the longitudinal force is generated by spin. But the roller is free to rotate in bearings, so this force shall only be such that the drag in the bearings and rolling resistance is overcome. Hence, we expect the rollers to rotate by a different angle compared to the previous experiment, and we denote by $\Delta \alpha_{\mathrm{NH}}$ the remaining rotation angle of the roller to reach the " 0 " mark, when the axle is rotated by the same number of turns as in the first experiment.

Actually, because the sharp end of the patch is directed outside the shield, we expect the roller to rotate more in the second experiment than in the first one. Indeed, after executing exactly 4 turns of the axle, the measured angle is $\Delta \alpha_{\mathrm{NH}}=0.594 \mathrm{rad}$.

The angles $\Delta \alpha_{H}$ and $\Delta \alpha_{N H}$ enable the first, qualitative comparison of elliptical and nonelliptical contacts. A big difference in angles $\Delta \alpha_{H}$ and $\Delta \alpha_{N H}$ indicates that spin can generate a substantial longitudinal force. If this phenomenon were absent, then the angles $\Delta \alpha_{\mathrm{H}}, \Delta \alpha_{\mathrm{NH}}$ would be equal, which is not the case.

Now we further rotate the axle by the angle $\Delta \beta$ and record the angle $\Delta \alpha_{0}$ by which the roller overpasses " 0 " mark. By doing this we recover the angular distance lost by the roller thus compensating the drag opposing the roller rotation.

The reading, after 4 turns gives $\Delta \alpha_{0}=0.990 \mathrm{rad}$.

The longitudinal creepage induced on the roller by the longitudinal creep force generated by spin is obtained as:

$$
v_{x}=1-\frac{r \omega}{R \Omega}=-\frac{r}{2 \pi N_{r} R} \Delta \alpha_{0}
$$

where $\mathrm{N}_{\mathrm{r}}$ (an even integer) is the number of turns of the axle.

It is easy to verify that $\Delta \alpha_{0}=\Delta \alpha_{\mathrm{H}}-\Delta \alpha_{\mathrm{NH}}$. The longitudinal creepage can be also determined by the equivalent formula

$$
v_{x}=-\frac{r}{2 \pi N_{r} R}\left(\Delta \alpha_{H}-\Delta \alpha_{N H}\right)
$$

The longitudinal creepage calculated according to formula (25) or (26) refers to the situation of no drag opposing the roller rotation. As such it facilitates theoretical interpretation of the experiment.

Using the measured values from the experiment and the dimensions of the apparatus reported above, the value of the longitudinal creepage $v_{x}=-0.0087$ is obtained. 
The experiment proves that spin can generate a substantial longitudinal creep force, because the induced longitudinal creepage is not small.

\section{CONCLUSIONS}

The extended Kalker book of tables KBTNH presented in this paper provides a fast and accurate method for determination of creep forces in non-Hertzian contact of wheel and rail and is able to provide the creep forces together with the moment for a given size and shape of the contact patch and for a given creepage condition.

The creep forces and moment in the contact patch provided by KBTNH have been compared with those computed directly by program CONTACT.

The comparison shows that results obtained by KBTNH and CONTACT are in good agreement. Discrepancies between KBTNH and CONTACT are only due to the applied discretisation of independent variables of KBTHN. For realistic combined creepage and spin, such as those shown in Section 6, the discrepancies are actually very low. Comparisons with FASTSIM algorithm for elliptical contact patches and SDEC regions showed high discrepancies between CONTACT and FASTSIM solutions for pure spin. The FASTSIM algorithm used by K-P method [5] does not predict creep forces and moment with sufficient accuracy under the same condition, which was one of our motivations to build KBTNH.

The very small computational effort required to interpolate KBTNH, combined with the good accuracy in determining creep forces and moment for non-Hertzian wheel-rail contact make the proposed method a suitable tool for MBS software designed to perform numerical simulation of rail vehicle dynamics.

An example of use of KBTNH in the context of multi-body simulation of rail vehicle dynamics was presented for the case of a freight wagon negotiating a curve. This simulation case was used to assess the contribution of the moment to the frictional work dissipated at wheel/rail contact on all wheels of the bogie.

Finally, the experiment described in Section 8 proves that spin can generate substantial longitudinal force in a non-elliptical, asymmetric contact patch.

\section{ACKNOWLEDGEMENTS}

- The authors wish to thank Prof. Piotr Skawinski of TU Warsaw for supervising the CNC machining of the shield and Ing. Janusz Kostro, MSc for his contribution into the axle unit design and realisation.

- Binbin Liu wishes to thank Associazione Eugenio e Germana Parizzi for providing financial support to his research.

\section{REFERENCES}

[1] Piotrowski, J., Liu, B., Bruni, S., The Kalker book of tables for non-Hertzian contact of wheel and rail, Vehicle System Dynamics, DOI:10.1080/00423114.2017.1291980, 2017.

[2] Vollebregt EAH. User guide for CONTACT, Rolling and sliding contact with friction. Technical report TR09-03, version 15.1, VORtech BV, Delft, The Netherlands; 2015. Available from:www.kalkersoftware.org. 
[3] Alonso, A., Giménez, J.G., Martín, L. M., Spin moment calculation and its importance in railway dynamics, Proceedings of the Institution of Mechanical Engineers, Part F: Journal of Rail and Rapid Transit. 223: 453-460, 2009.

[4] Birger, I.A., Panovko, J.G. (Editors): Procsnost, ustoicivost, kolebaniya. (Strength, stability, vibrations). A handbook in Russian, Vol. 2, Chap.11. Mashinostroyeniye, Moscow 1968.

[5] Piotrowski, J., Kik, W., A simplified model of wheel/rail contact mechanics for nonHertzian problems and its application in rail vehicle dynamic simulations. Vehicle System Dynamics. 46(1-2), 27-48, 2008.

[6] Piotrowski, J., Steady-state curving of railway bogie with damper-coupled wheelsets. Evaluation in terms of non-linear curving theory. Archives of Transport, volume 2, issue 3, 1990 . 


\section{List of captions of figures}

Figure 1: The SDEC contact region. $\mathrm{x}$ - rolling direction, $\mathrm{y}$ - lateral direction.

Figure 2: The normal pressure distribution for SDEC contact region: (a) contact patch, (b) normal pressure profile, and (c) normal pressure distribution.

Figure 3: The normalised moment as a function of $\operatorname{SDEC} \psi$ calculated by different methods for $v_{\mathrm{x}}=$ $v_{\mathrm{y}}=0, \varphi=1.0 \mathrm{~m}^{-1}$, and $\delta=0.1 \mathrm{~mm}$, for (a) the first and (b) second comparison, respectively.

Figure 4: The normalised moment as a function of spin $\varphi$ calculated by different methods with $\psi=0.4, v_{\mathrm{x}}=v_{\mathrm{y}}=0$, and $\delta=0.1 \mathrm{~mm}$, for (a) the first and (b) second comparison, respectively.

Figure 5: The normalised moment as a function of spin $\varphi$ calculated by different methods with $\psi=0.8$ for $v_{\mathrm{x}}=v_{\mathrm{y}}=0$, and $\delta=0.1 \mathrm{~mm}$ for (a) the first and (b) second comparison, respectively.

Figure 6: Non-dimensional creep forces obtained by KBTNH versus CONTACT.

Figure 7: The normalised (a1, a2) longitudinal, (b1, b2) lateral creep force and (c1, c2) moment as a function of modulus of creepage for $\mathrm{g}=0.5, \alpha=\pi / 4, \psi=0.8$ (left column$\mathrm{KBTNH})$ and $\psi=0$ (right column-elliptical KBTH).

Figure 8: The sign convention for wheelset forces [6] $\left(\mathrm{u}_{\mathrm{ij}}\right.$ is forward shift of the contact point due to yaw).

Figure 9: (a) The drawing of the apparatus and (b) its photograph. 